\title{
Application of Resorbable Poly(Lactide-co-Glycolide) with Entangled Hyaluronic Acid as an Autograft Extender for Posterolateral Intertransverse Lumbar Fusion in Rabbits
}

\author{
William R. Walsh, Ph.D.,' Rema A. Oliver, Ph.D.,' Gary Gage, B.S., ${ }^{1}$ Yan Yu, Ph.D., ${ }^{1}$ David Bell, MBBS, \\ Jeremy Bellemore, M.S., ${ }^{1}$ and Huston Davis Adkisson, Ph.D. ${ }^{2}$
}

Facilitating fusion between bony segments in a reliable and reproducible manner using a synthetic bone graft material has a number of benefits for the surgeon as well as the patient. Although autograft remains the gold standard, associated comorbidities continue to drive the development of new biomaterials for use in spinal fusion. The ability of autograft alone and autograft combined with a radiolucent biomaterial composed of resorbable osteoconductive poly(lactide-co-glycolide) with entangled hyaluronic acid to facilitate fusion was examined in a single-level noninstrumented posterolateral intertransverse lumbar fusion model in New Zealand White rabbits. Progressive bone formation was demonstrated radiographically for the extender group (synthetic biomaterial plus autograft) between 3 and 6 months. Computed tomography revealed a new cortical shell in the fusion mass at 3 and 6 months for both study groups. Tensile testing at 6 months demonstrated that the quality of bone formed between the intertransverse space was equivalent for both study groups. Histologic evaluation of the fusion mass revealed new bone on and adjacent to the transverse processes with the synthetic biomaterial group that extended laterally, supporting the osteoconductive nature of the material. Histological evidence of endochondral bone growth in the intertransverse space was observed for the autograft plus synthetic biomaterial group. Bone remodeling, new marrow spaces, and peripheral cortices were observed for each study group at 3 months that matured by 6 months. These findings support the use of a radiolucent biosynthetic material comprising poly(lactide-co-glycolide) with integrated hyaluronic acid as an autograft extender for lumbar intertransverse fusion.

\section{Introduction}

$\mathbf{F}$ ACILITATING FUSION BETWEen bony segments in a reliable and reproducible manner using a synthetic bone graft material has a number of benefits for the surgeon as well as the patient. Although bone autograft remains the gold standard, associated comorbidities and limitations of supply, particularly in the case of multilevel fusion, continue to drive developments of new biomaterials for use in spinal fusion. The presence of inorganic components in many graft materials and their slow resorption profiles often make radiographic assessment of the fusion mass subjective and difficult.

This study evaluated the preclinical effectiveness of a novel biomaterial composed of resorbable osteoconductive poly(lactide-co-glycolide) (PLGA) with entangled hyaluronic acid (HyA) for use in spine fusion when applied as an au- tograft extender. HyA, a widely distributed polysaccharide component of the extracellular matrix of connective tissues and bone marrow, has been reported to play an important role in tissue repair and regeneration. ${ }^{1-4}$ The scientific rationale underlying the development of the current material for bone repair stems from the need for a compressive resistant osteoconductive scaffold with improved biologic properties and a controlled rate of resorption reflective of normal bone turnover. The entanglement of HyA within the osteoconductive biomaterial increases the hygroscopic properties of the polymer backbone, ${ }^{5}$ facilitating cell migration and attachment. ${ }^{3,6-8}$ In light of the roles ascribed to HyA during embryonic development and tissue repair, this polysaccharide, whose chemical structure remains highly conserved among species, may be the best candidate molecule for integration within the hydrophobic PLGA backbone. Previous studies have demonstrated the ability of InQu to

\footnotetext{
${ }^{1}$ Surgical and Orthopaedic Research Laboratories, University of New South Wales, Prince of Wales Hospital, Randwick, Australia.

${ }^{2}$ ISTO Technologies, Inc., St. Louis, Missouri.
} 
support rapid bone formation in contained metaphyseal defects (cancellous bone) of adult rabbits via endochondral ossification. $^{6}$

The present study assessed the in vivo response of a novel PLGA/HyA hybrid scaffold as an autograft extender in a standardized model of posterolateral intertransverse lumbar fusion in adult New Zealand White rabbits. ${ }^{9-12}$ Radiographic and histological outcomes were evaluated at 3 and 6 months after surgery, with a subset of the 6-month animals designated for assessment of bone quality via biomechanical testing of the fusion mass to failure.

\section{Materials and Methods}

\section{Animal model}

A single-level noninstrumented posterolateral spinal fusion was performed (bilaterally) in 26 New Zealand White rabbits (6 months old) after institutional ethical clearance. The surgical model is based on the original work of Boden and coworkers ${ }^{9}$ as outlined in detail by Palumbo and colleagues. ${ }^{10,11}$ The following groups were examined in this study: autograft alone and InQu combined with autograft (50:50), also referred to as the InQu extender group (Table 1). Six rabbits per group were harvested at 3 months and seven animals per group were harvested at 6 months for assessment of spine fusion via radiography ( $\mathrm{X}$-rays and computed tomography [CT]) and histology. A subset of the 6- month animals (four per group) were used for biomechanical testing.

\section{Characterization of InQu granules}

PLGA (75:25) and HyA were combined at a ratio of 9:1. Resulting bulk material was sized in a grinding mill to produce granules ranging from 2 to $5 \mathrm{~mm}$ in the longest dimension. InQu granules were e-beam sterilized before use. The porous nature of the InQu material was evaluated using an environmental electron microscope (Hitachi TM 1000) as well as an FEI Siron SEM with Au sputter coating. Images were evaluated at different magnifications to examine the characteristics of the material and pore size distribution.

\section{Surgical procedure}

Animals were anesthetized for surgery using isoflurane/ oxygen inhalation. A midline incision was made in the skin and the intermuscular plane between the multifidus and longissimus muscles was bluntly developed to expose the L5 and L6 transverse processes (TPs). A high-speed burr (Midas Rex; Medtronic) with saline irrigation was used to decorticate the TPs in a uniform and reproducible manner in all animals $10 \mathrm{~mm}$ from the base of the TPs as well as along the vertebral body. Corticocancellous autograft was harvested from the iliac crest and morcelized into small pieces using a ronguer and either used alone or mixed with InQu (50:50)

Table 1. Study Design

\begin{tabular}{lcc}
\hline & \multicolumn{2}{c}{ Sample size } \\
\cline { 2 - 3 } Groups & 3 months & 6 months \\
\hline InQu + autograft (50:50) & 6 & 7 \\
Autograft & 6 & 7 \\
\hline
\end{tabular}

(Fig. 1). The same materials were placed on the decorticated TPs adjacent to the vertebral body bilaterally (2 cc per side) as per the study design.

\section{Radiographic assessment of fusion}

The general integrity of the incision was noted, along with any evidence of macroscopic reaction of the underlying subcutaneous tissues after euthanasia, at 3 and 6 months. Faxitron radiographs of the harvested spines were taken (Faxitron $\mathrm{HP}$ ) in the anteroposterior orientation using digital plates (Agfa). DICOM data were viewed using DICOMWorks v1.3.5 and graded in a blinded fashion to assess bony fusion between the TPs on each side of the vertebral body. Left and right sides of the fusion mass were individually scored as fused (1) or not fused (0). A second radiographic score illustrating the quality of bone formed based on percent fill was assigned to the left and right sides by two blinded assessors. A numerical grade ranging from 0 to 5 was used where a score of 0 represented $<10 \%$ bone fill; 1 represented $11 \%-20 \%$ bone fill; 2 represented $21 \%-40 \%$ bone fill; 3 represented $41 \%-60 \%$ bone fill; 4 represented $61 \%-80 \%$ bone fill; and 5 represented $81 \%-$ $100 \%$ bone fill. Radiographic grading was evaluated statistically using a two-tailed Fisher's Exact test.

Each spine was examined using a clinical CT scanner (Toshiba Asteion). Images were taken in the axial plane at a slice thickness of $0.5 \mathrm{~mm}$ using $100 \mathrm{kV}$ and $60 \mathrm{mAs}$. CT images in DICOM format were imported and analyzed with MIMICS software (Version 11; Materialise). Threedimensional (3D) models were created to observe the fusion masses between the TPs using a standardized minimum threshold of 226 Hounsfield Units. The fusions were evaluated in the axial, coronal, and sagittal planes in the raw CT images to assess the presence of residual material, new bone, and continuity of fusions between the TPs.

\section{Biomechanical assessment of new bone quality}

The biomechanical properties were examined in a quantitative fashion using destructive mechanical testing in tension at 6 months using established techniques. ${ }^{9,12}$ Samples were carefully dissected so that only the fusion mass participated to resist the tensile load. The disc, facet joints, intertransverse membrane, and posterior and supratransverse ligaments were sharply dissected using a \#15 scalpel blade before loading on the testing machine. Uniaxial tension was applied at a constant rate of $5 \mathrm{~mm} / \mathrm{min}^{13,14}$ using a testing jig that allowed two degrees of freedom to minimize off-axis
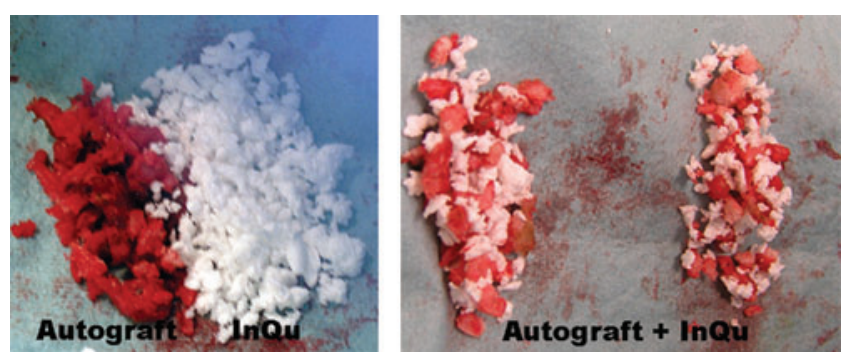

FIG. 1. InQu granules were combined with autograft harvested from the iliac crest and morcelized to extend the local autograft (50:50 by volume). Color images available online at www.liebertonline.com/ten. 
loading and apply uniaxial tension. ${ }^{12}$ Spines were fixed in the testing rig using a $3.2 \mathrm{~mm}$ stainless steel pin drilled through each vertebra in the anteroposterior direction and attached to an MTS 858 Bionix testing machine (MTS) and a $2 \mathrm{kN}$ load cell. Samples were fixed in the machine while in load control to avoid any preloading before testing. Load/ displacement data were continually recorded at a rate of $100 \mathrm{~Hz}$ and the peak load, stiffness, and energy to peak load were calculated for each specimen using an in-house MatLab program (MatLab). Mechanical data were analyzed using a one-way analysis of variance followed by a Games Howell post-hoc test (SPSS Version 17).

\section{Histological processing}

Dissected tissues were processed for routine paraffin embedding by fixation in cold phosphate buffered formalin solution for a minimum of $48 \mathrm{~h}$ and decalcified in $10 \%$ formic acid-phosphate buffered formalin solution. All spines were processed for histology in this study. The left-hand side of the fusion was sectioned in the coronal plane, whereas the right-hand side was sectioned in the sagittal plane after decalcification. Two coronal blocks (anterior and posterior) and four sagittal blocks (midline to lateral) were obtained for each animal. Five micrometer sections cut from each block using a Leica microtome (Leica Microsystems $\mathrm{GmbH}$ ) were stained with hematoxylin and eosin, tetrachrome for blinded examination of new bone formation and host-graft material interactions with the adjacent bony beds of the TPs and vertebral body, as well as midway between the TPs with the muscle on the dorsal aspect and the intertransverse membranes on the ventral aspect. ${ }^{12}$

\section{Results}

The porous 3D nature of the synthetic biomaterial ( $\mathrm{InQu}$ ) was readily apparent upon electron microscopic evaluation in which the porosity within the material was observed to range from $75 \%$ to $90 \%$, displaying a range of pore sizes $(\sim 1-300 \mu \mathrm{m})$ based on electron microscopy (Fig. 2). InQu combined well with iliac crest autograft as an extender, which was easily placed into the surgical site and readily adsorbed local blood. No gross evidence of the InQu material was noted upon harvest during dissections in the fascial or soft tissue planes at 3 or 6 months after surgery, and there was no evidence of infection at the fusion site for either study group at each time point.

Posteroanterior Faxitron radiographs revealed a progression in bone formation with time between 3 and 6 months for both the autograft and extender groups (Fig. 3). New bone formation was observed between the TPs in greater volumer than found in decorticated controls using this rabbit model. ${ }^{12}$ Continuous fusion at the operated level was graded blinded by two observers. Six animals in each group at 3 months provided 12 sites for grading per group (6 right and 6 left), whereas seven animals in each group at 6 months provided 14 sites for grading per group (7 right and 7 left). Table 2a presents a summary of the number of sites demonstrating continuous fusion as determined radiographically for each group studied at each endpoint while Table $2 \mathrm{~b}$ represents the quality of bone formed based on the radiographic assessment of percent fill. The fusion rate achieved for iliac crest autograft at 3 months was $91.7 \%$ and that of the InQu ex-

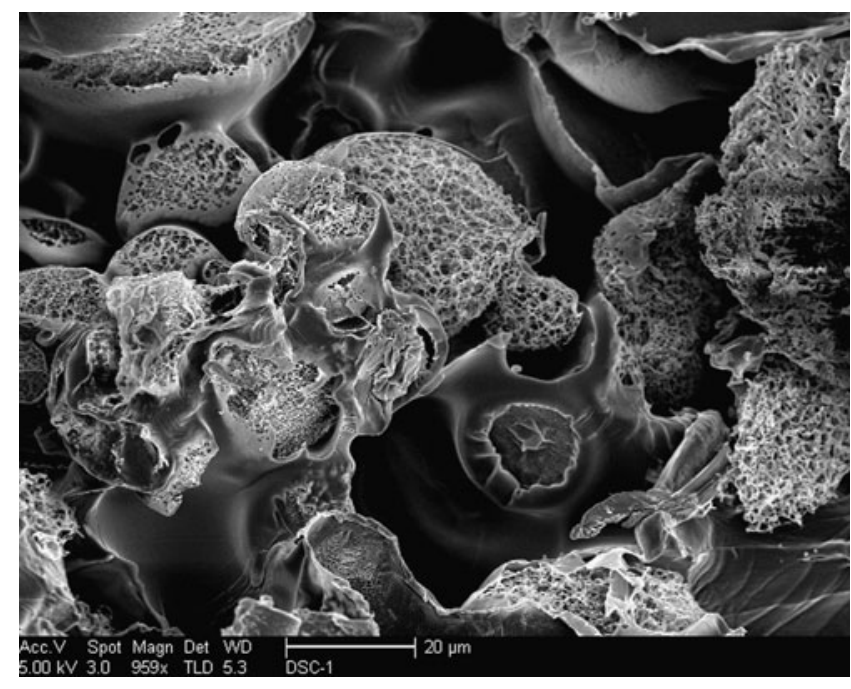

FIG. 2. Scanning electron microscopy of the InQu granules revealed macroscopic as well as microscopic porous domains within the material available for cellular integration. The porosity within the material ranged from $75 \%$ to $90 \%$ with a range of pore sizes $(\sim 1-300 \mu \mathrm{m})$.

tender group was $66.7 \%$. Despite an apparent difference in radiographic grading between groups, no statistically significant differences in union rates were detected at 3 and 6 months $(p>0.05)$. CT revealed similar results to the radiographic findings in terms of continuous fusion between the TPs. Sagittal CT views provided evidence of a new cortical shell and marrow spaces in the fusion mass at 3 and 6 months (Fig. 4) for both InQu extender and autograft alone.

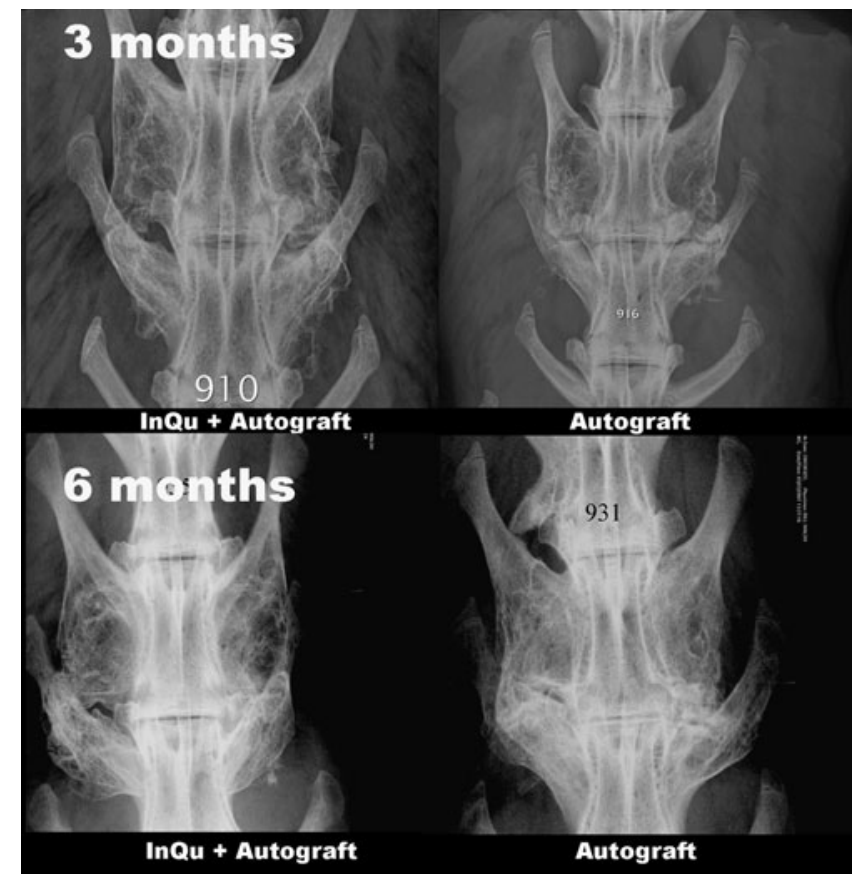

FIG. 3. Posteroanterior Faxitron radiographs at 3 and 6 months revealed a progression in healing with time in both groups. New bone formation was observed on the transverse processes above that found in historical decorticated controls using this rabbit model. 
Table 2. Radiographic Grading of Fusion and Semiquantitative Assessment of Bone Fill at Fusion Level (L5-L6)

\begin{tabular}{lcccc}
\hline & \multicolumn{3}{c}{ a. Radiographic grading of fusion ${ }^{\mathrm{a}}$} \\
\cline { 2 - 5 } Groups & \multicolumn{2}{c}{3 months } & \multicolumn{1}{c}{6 months } \\
\cline { 2 - 5 } InQu + autograft (50:50) & Fused & Not fused & Fused & Not fused \\
Autograft & 8 & 4 & 10 & 4 \\
\hline
\end{tabular}

\begin{tabular}{llcll}
\hline & \multicolumn{3}{c}{ b. Semiquantitative assessment of bone fill } \\
\cline { 2 - 5 } Groups & \multicolumn{2}{c}{3 months } & & 6 months \\
\cline { 2 - 5 } InQu + autograft (50:50) & Bone left & Bone right & Bone left & Bone right \\
Autograft & $3.83(1.17)$ & $4.17(0.75)$ & $4.29(0.91)$ & $4.71(0.47)$ \\
\cline { 2 - 5 } & $5.00(0)$ & $4.5(0.84)$ & $4.57(0.76)$ & $4.71(0.73)$
\end{tabular}

The six animals in each group at 3 months provided 12 sites for grading per group ( 6 right and 6 left), whereas seven animals in each group at 6 months provided 14 sites for grading per group (7 right and 7 left).

${ }^{a}$ The radiographic grading improved with time between 3 and 6 months but did not change within groups $(p>0.05)$.

${ }^{\mathrm{b}}$ Similar results were found based on the percentages of new bone observed radiographically at 3 and 6 months for each group.

FIG. 4. Computed tomography data were analyzed using MIMICS and revealed similar results to the radiographic findings in terms of continuous fusion between the transverse processes. Sagittal views demonstrated the presence of a new cortical shell and marrow spaces in the fusion mass at 3 and 6 months for the InQu extender and autograft alone.

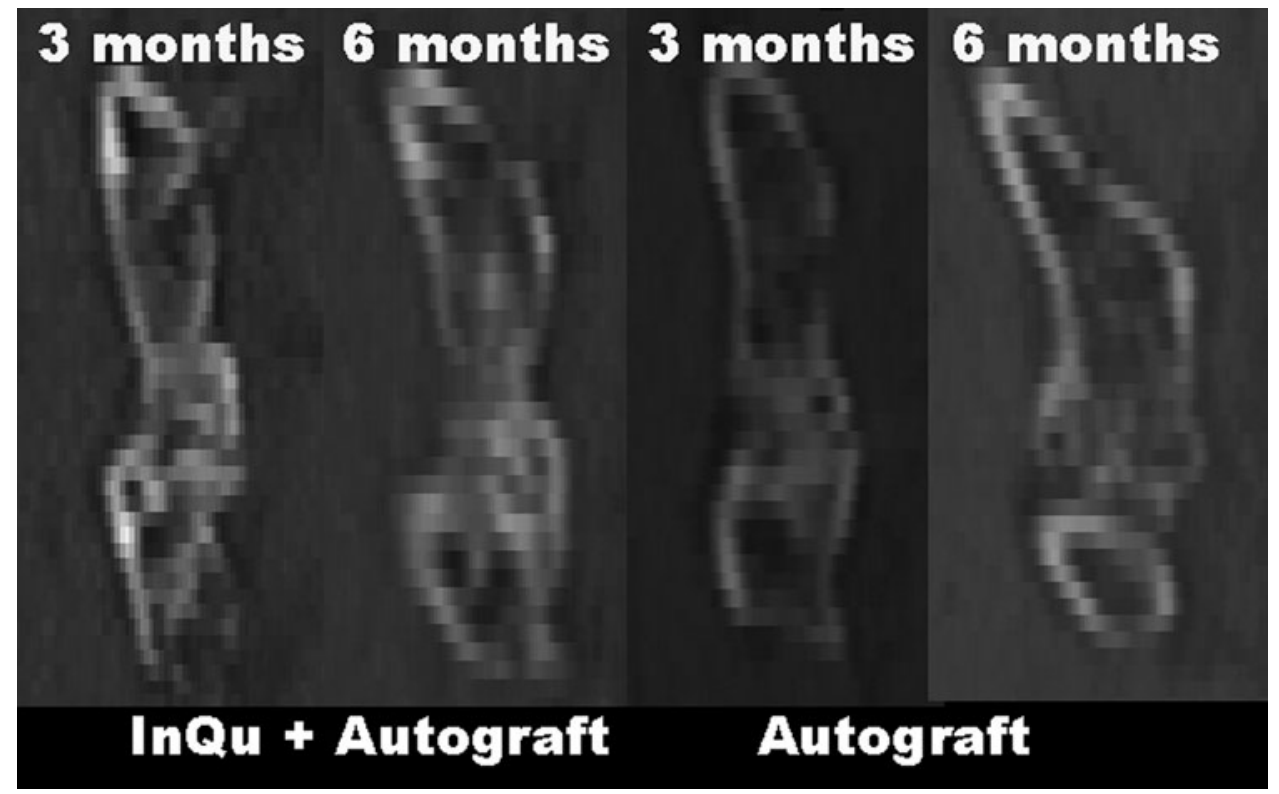

Table 3. Mechanical Assessment of Bone Quality

\begin{tabular}{lccr}
\hline Group & Peak load $(\mathrm{N})$ & Stiffness $(\mathrm{N} / \mathrm{mm})$ & Energy $(\mathrm{N} / \mathrm{mm})$ \\
\hline InQu + autograft (50:50) & $227.87 \pm 33.01$ & $113.63 \pm 10.24$ & $371.35 \pm 206.58$ \\
Autograft & $249.20 \pm 84.66$ & $128.32 \pm 59.79$ & $387.27 \pm 104.81$ \\
\hline
\end{tabular}

The tensile properties at 6 months in the autograft group were not statistically different compared to the autograft combined with InQu (50/50). 

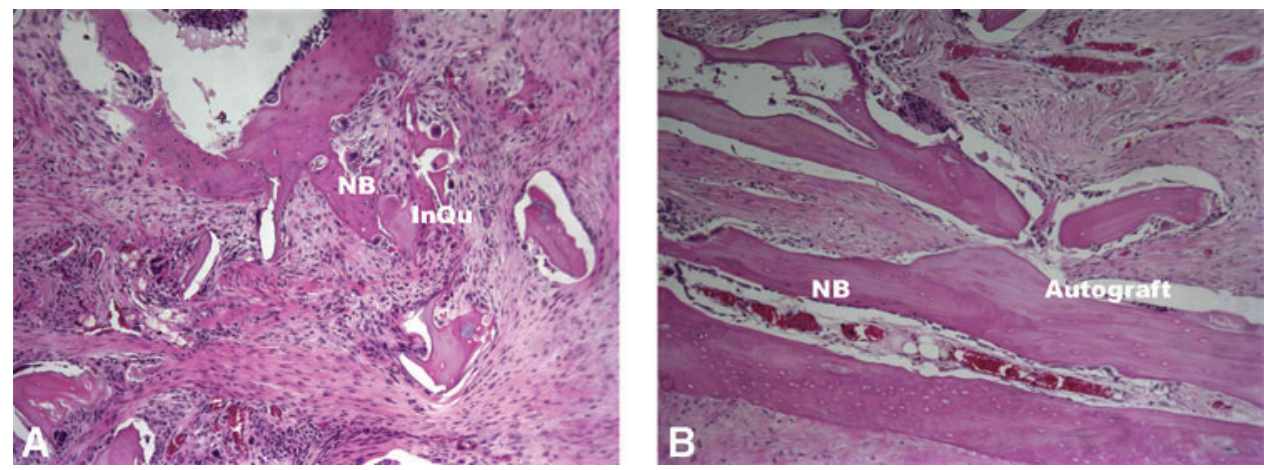

FIG. 5. (A) H \& E, 10× objective; the histological response of InQu + Autograft (A) and Autograft alone (B) was similar at 3 months demonstrating normal bone healing at the interface of the decorticated surfaces and bone remodeling. Both treatments demonstrated areas of new bone formation (NB) and marrow spaces. Residual material (InQu) was present at 3 months (A).

Uniaxial tensile testing of the fusion mass to failure at 6 months revealed that the extender group was equivalent to autograft (Table 3). Mean peak tensile loads measured for the autograft group $(249.2 \pm 84.7 \mathrm{~N})$ were not statistically different from those measured for the extender group $(227.9 \pm$ $33.0 \mathrm{~N}$ ). Similarly, the stiffness and energy (Table 3) of the fusion masses measured for each study group did not differ at 6 months.

Sagittal and coronal histology provided a review of the in vivo biologic response of host tissues to InQu extender and corticocancellous autograft in two separate planes after placement of study materials in the posterolateral gutter. The histological response of both groups was similar at 3 and 6 months, demonstrating normal bone healing at the interface of the decorticated surfaces and laterally between the TPs. Bone remodeling progressed from 3 to 6 months, with no evidence of corticocancellous autograft at the final endpoint. Both treatments revealed areas of new bone formation on remaining autograft at 3 months, along with the presence of residual InQu material in the extender group (Fig. 5). New marrow spaces and peripheral cortices were observed for the InQu extender and autograft groups at 3 months that matured by 6 months, as demonstrated in the sagittal plane (Fig. 6). No histological evidence of the InQu material was found at 6 months. The combination of InQu with autograft did not alter the resorption of autograft and was equivalent to autograft alone based on histological findings at 3 and 6 months. Endochondral bone formation was observed for the extender group where calcified cartilage was found to undergo replacement by woven bone upon vascularization of the cartilage intermediate. No overt inflammation or presence of giant cells was observed in association with the PLGA backbone of the extender group at either time point.

\section{Discussion}

This study evaluated the in vivo performance of InQu, a novel synthetic bone graft substitute comprising PLGA with entangled HyA, to promote bilateral posterolateral intertransverse process fusion of the rabbit spine, when used as an extender after combination (50:50) with corticocancellous bone autograft. The material was porous and combined well with local graft and blood as well as being radiolucent. A control group consisting of the gold-standard iliac crest autograft was used for comparison. This well-characterized animal model is commonly used to evaluate osteoinductive bone graft materials ${ }^{15,16}$ and osteoconductive materials, including allograft bone, ${ }^{17}$ and collagen-mineral composites. ${ }^{12}$ The present study demonstrated that resorbable osteoconductive PLGA, containing integrated HyA and lacking an inorganic mineral component, is capable of extending autograft to achieve fusion rates equivalent to that of autograft at 3 and 6 months postoperatively in the Boden model.

The local presence of HyA coupled with the polymer matrix was shown to be biocompatible, supporting new bone formation via endochondral ossification. Bone formed between the TPs remodeled laterally to yield a cortex containing trabecular bone as demonstrated histologically at 3 and 6 months. The PLGA component appeared to be well tolerated and fully resorbed in this model by 6 months. A 50:50 mixture of InQu and autograft was chosen to represent
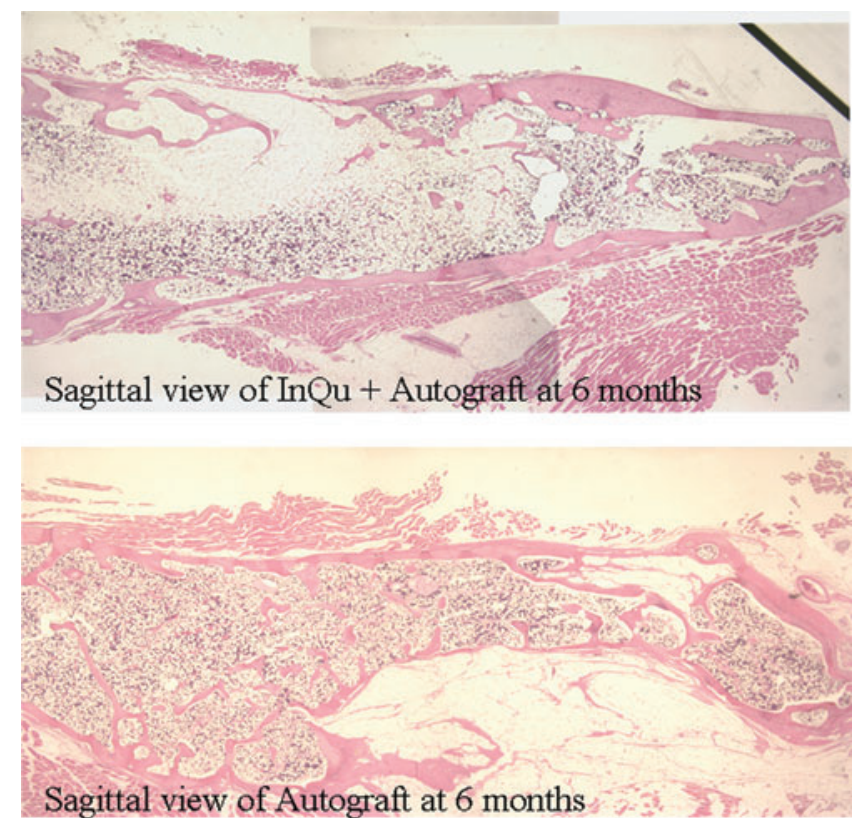

FIG. 6. Sagittal section, hematoxylin and eosin, 1.25x objective. No evidence of the InQu material or autograft was found at 6 months. Both treatment groups demonstrated a mature peripheral cortex and normal bone marrow spaces at 6 months. 
a reasonable clinical use of this material as an autograft extender. However, clinical use will likely involve the harvest of local bone to eliminate those risks associated with iliac crest harvest.

The combined use of synthetic and naturally occurring polymers is a common strategy used in the production of novel biomaterials that are intelligent and capable of interacting specifically with their biologic environment that is both hygroscopic and conductive. ${ }^{18}$ The $3 \mathrm{D}$ bulking properties of PLGA create a compressive resistant template for cell adhesion, whereas HyA appears to provide a biologic environment conducive to bone formation. HyA, or hyaluronan, is well recognized to play an integral role in tissue morphogenesis and repair, mediating cellular migration and adhesion during wound healing. ${ }^{1-4,7}$ Additionally, HyA is reported to support osteogenic differentiation of progenitor cells in vitro. ${ }^{19,20}$ A role for HyA in bone healing in vivo was first reported by Sasaki and Watanabe, where injection of high-molecularweight HyA into the bone marrow cavity of rats subsequent to ablation injury was found to accelerate formation of new bone at the periosteal and endosteal surfaces. ${ }^{21}$ Additionally, it was found that application of high-molecular-weight HyA to excavated marrow cavities supported repopulation of marrow elements displaying a normal complement of cells in less time when compared to sham controls. ${ }^{21}$

While the mechanism underlying the positive effect of HyA on bone wound healing remains unclear, it is known that HyA interacts directly with target cells involved in bone formation via cell surface binding of CD44, including osteoand endothelial-progenitor cells, osteoblasts, and osteoclasts, ${ }^{22-25}$ and that oligosaccharides produced by enzymatic digestion of high-molecular-weight HyA support angiogenesis. ${ }^{26}$ There is scientific evidence to suggest that HyA may function also to sequester bioactive growth factors within the extracellular matrix of tissues, protecting these proteins from rapid cleavage and inactivation. ${ }^{27,28}$ In this way, bioactive growth factors may be locally concentrated, serving as a depot to induce the proliferation and differentiation of resident progenitor cells as required for wound repair. Locci and coworkers have shown that transforming growth factor beta (TGF- $\beta$ ) within TGF- $\beta$-HyA complexes is protected from tryptic degradation in vitro ${ }^{27}$ and that scaffolds created from esterified HyA efficiently bind and release TGF- $\beta$ to enhance matrix formation by human osteoblasts in vitro. ${ }^{29}$ This drug release mechanism can be applied to bone and cartilage repair where HyA-based scaffolds loaded with recombinant human bone morphogenetic protein-2 were shown to release low levels of bioactive protein over an extended period as compared to that of nonporous hydrogels prepared from type I collagen. ${ }^{30}$

Chemically modified HyA has been studied extensively in applications of tissue engineering for the repair of cartilage and bone. HyA-based scaffolds are reported to allow faster progenitor cell recruitment within treated defects, resulting in rapid tissue healing. ${ }^{8,31}$ Solchaga and coworkers ${ }^{31}$ compared bone healing in rabbits after treatment with HyAbased scaffolds and PLGA alone, demonstrating a delay in bone formation in the case of PLGA. The authors hypothesized that the release of biologically active hyaluronan oligomers early in the repair process may contribute to or trigger rapid endochondral bone growth after treatment of osteochondral defects in rabbits. ${ }^{31}$ Certainly, the present his- tological results and previous findings support the conclusion that HyA may actively participate in the regeneration of bone. ${ }^{4,6,8,21,31,32}$ Using the same model employed in the present study, Yee et al. reported that demineralized bone allograft combined with HyA hydrogel enhanced both the quantity and quality of new bone formed in the intertransverse spaces when used as an adjunct to autogenous

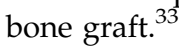

HyA has a proven history of safe use in the medical device industry where it is used as a surgical aid in ophthalmology and as a therapeutic agent in joint disease and wound healing. Most notably, because HyA is a polysaccharide whose chemical structure is conserved between bacterial and mammalian sources, it is not reported to generate an antigenic response that would abrogate wound healing.

The New Zealand White rabbit Boden model is a useful preclinical scenario to evaluate biomaterials before human clinical use, yielding similar nonunion rates ranging from $30 \%$ to $40 \%$. ${ }^{9,34}$ Despite several previous studies having validated the Boden model for spinal fusion, it is not without limitations and does not completely mimic the human clinical scenario considering differences in geometry, biomechanics, and ambulation. Therefore, one must cautiously extrapolate the results of the current rabbit study into clinical practice. Yee et al. $^{35}$ reported previously that fine detail radiography may be inadequate for the identification of nonunions in the Boden model. For this reason, manual palpation and CT have been advocated as an adjunct to radiographic follow-up when using this model. Threedimensional reconstructed CT images were used in the present study to document continuity of bone formed between the TPs. With regard to radiographic rates of fusion, we feel that the observed trend of a decrease in scores between 3 and 6 months for the autograft control group may reflect the remodeling of residual autograft that was confirmed histologically to be present at 3 months and subsequently resorbed by 6 months, and that this may have overestimated the 3 month fusion rates for the autograft control group. Other investigators have reported control fusion rates of between $50 \%$ and $66 \%$ for cancellous autograft using manual palpation. ${ }^{9,34,35}$ Consequently, in the absence of clinical data (e.g., palpation in the case of rabbits), we must rely on the mechanical measurement of bone quality estimated for each study group at 6 months. While no statistically significant differences in each of the mechanical measurements (e.g., stiffness, peak load, and energy) were noted between study groups, the variability in these measurements were two to fivefold greater for the autograft control group versus InQu extender. This finding suggests that bone formed in association with the synthetic osteoconductive scaffold may have integrated more consistently with host bone at the interface of the decorticated TPs than morcelized corticocancellous autograft. Another potential weakness of the study is low sample size and reduced power to detect any differences between autograft and the autograft plus InQu (extender) group. Based on the means and standard deviations, a sample size of $n=100$ per group would have been required to achieve a power of 0.75 , which is unrealistic. We did not include any decorticated control samples where no graft material was placed into the posterolateral space. We have examined this group previously using the same animal model and endpoints. ${ }^{12}$ 
In summary, a novel radiolucent osteobiologic scaffold comprising PLGA with integrated HyA successfully supported new bone formation when applied as an autograft extender in a rabbit model for posterolateral intertransverse lumbar fusion. Equivalence in fusion rates and bone quality were demonstrated at 6 months for InQu plus autograft (extender) when compared to cancellous autograft alone. These observations support the use of InQu bone graft extender for posterolateral lumbar fusion in humans and may reduce the requirement for iliac crest harvest where sufficient local bone is available.

\section{Disclosure Statement}

This study was performed by financial support provided by ISTO Technologies. Gary Gage and H. Davis Adkisson are paid employees of ISTO Technologies.

\section{References}

1. Caplan, A.I. Tissue engineering designs for the future: new logics, old molecules. Tissue Eng 6, 1, 2000.

2. Chen, W.Y., and Abatangelo, G. Functions of hyaluronan in wound repair. Wound Repair Regen 7, 79, 1999.

3. Knudson, C.B., and Knudson, W. Hyaluronan-binding proteins in development, tissue homeostasis, and disease. FASEB J 7, 1233, 1993.

4. Weigel, P.H., Fuller, G.M., and LeBoeuf, R.D. A model for the role of hyaluronic acid and fibrin in the early events during the inflammatory response and wound healing. J Theor Biol 119, 219, 1986.

5. Lee, C.T., and Lee, Y.D. Preparation of porous biodegradable poly(lactide-co-glycolide)/hyaluronic acid blend scaffolds: characterization, in vitro cells culture and degradation behaviors. J Mater Sci Mater Med 17, 1411, 2006.

6. Adkisson, H.D., Liu, L., Alvarez, U., Abe, S., Matava, M., Hruska, K.A., Katwal, A., Kizer, N., and Seyedin, M. Accelerated bone and cartilage repair using a novel biomaterial scaffold. Abstract presented at the 53rd Annual Meeting of the Orthopaedic Research Society, 2007. Abstract no. 1513.

7. Lesley, J., Hascall, V.C., Tammi, M., and Hyman, R. Hyaluronan binding by cell surface CD44. J Biol Chem 275, 26967, 2000.

8. Solchaga, L.A., Temenoff, J.S., Gao, J., Mikos, A.G., Caplan, A.I., and Goldberg, V.M. Repair of osteochondral defects with hyaluronan- and polyester-based scaffolds. Osteoarthritis Cartilage 13, 297, 2005.

9. Boden, S.D., Schimandle, J.H., and Hutton, W.C. An experimental lumbar intertransverse process spinal fusion model. Radiographic, histologic, and biomechanical healing characteristics. Spine 20, 412, 1995.

10. Palumbo, M., Valdes, M., Robertson, A., Sheikh, S., and Lucas, P. Posterolateral intertransverse lumbar arthrodesis in the New Zealand White rabbit model: I. Surgical anatomy. Spine J 4, 287, 2004.

11. Valdes, M., Palumbo, M., Appel, A.J., McAllister, S., and Ehrlich, M. Posterolateral intertransverse lumbar arthrodesis in the New Zealand White rabbit model: II. Operative technique. Spine J 4, 293, 2004.

12. Walsh, W.R., Vizesi, F., Cornwall, G.B., Bell, D., Oliver, R., and $\mathrm{Yu}, \mathrm{Y}$. Posterolateral spinal fusion in a rabbit model using a collagen-mineral composite bone graft substitute. Eur Spine J 18, 1610, 2009.

13. Boden, S.D., Schimandle, J.H., and Hutton, W.C. Volvo Award in basic sciences. The use of an osteoinductive growth factor for lumbar spinal fusion. Part II: study of dose, carrier, and species. Spine 20, 2633, 1995.

14. Valdes, M., Moore, D.C., Palumbo, M., Lucas, P.R., Robertson, A., Appel, J., Ehrlich, M.G., and Keeping, H.S. rhBMP-6 stimulated osteoprogenitor cells enhance posterolateral spinal fusion in the New Zealand white rabbit. Spine J 7, 318, 2007.

15. Boden, S.D., Schimandle, J.H., Hutton, W.C., Damien, C.J., Benedict, J.J., Baranowski, C., and Collier, S. In vivo evaluation of a resorbable osteoinductive composite as a graft substitute for lumbar spinal fusion. J Spinal Disord 10, 1, 1997.

16. Damien, C.J., Grob, D., Boden, S.D., and Benedict, J.J. Purified bovine BMP extract and collagen for spine arthrodesis: preclinical safety and efficacy. Spine 27, S50, 2002.

17. Nugent, P.J., and Dawson, E.G. Intertransverse process lumbar arthrodesis with allogeneic fresh-frozen bone graft. Clin Orthop Relat Res 287, 107, 1993.

18. Sakiyama-Elbert, S.E., and Hubbell, J.A. Functional biomaterials: design of novel biomaterials. Annu Rev Mater Res 31, 183, 2001.

19. Huang, L., Cheng, Y.Y., Koo, P.L., Lee, K.M., Qin, L., Cheng, J.C., and Kumta, S.M. The effect of hyaluronan on osteoblast proliferation and differentiation in rat calvarial-derived cell cultures. J Biomed Mater Res A 66, 880, 2003.

20. Zou, L., Zou, X., Chen, L., Li, H., Mygind, T., Kassem, M., and Bunger, C. Effect of hyaluronan on osteogenic differentiation of porcine bone marrow stromal cells in vitro. J Orthop Res 26, 713, 2008.

21. Sasaki, T., and Watanabe, C. Stimulation of osteoinduction in bone wound healing by high-molecular hyaluronic acid. Bone 16, 9, 1995.

22. Noonan, K.J., Stevens, J.W., Tammi, R., Tammi, M., Hernandez, J.A., and Midura, R.J. Spatial distribution of CD44 and hyaluronan in the proximal tibia of the growing rat. J Orthop Res 14, 573, 1996.

23. Cao, J.J., Singleton, P.A., Majumdar, S., Boudignon, B., Burghardt, A., Kurimoto, P., Wronski, T.J., Bourguignon, L.Y., and Halloran, B.P. Hyaluronan increases RANKL expression in bone marrow stromal cells through CD44. J Bone Miner Res 20, 30, 2005.

24. Ariyoshi, W., Takahashi, T., Kanno, T., Ichimiya, H., Takano, H., Koseki, T., and Nishihara, T. Mechanisms involved in enhancement of osteoclast formation and function by low molecular weight hyaluronic acid. J Biol Chem 280, 18967, 2005.

25. Murphy, J.F., Lennon, F., Steele, C., Kelleher, D., Fitzgerald, D., and Long, A.C. Engagement of CD44 modulates cyclooxygenase induction, VEGF generation, and proliferation in human vascular endothelial cells. FASEB J 19, 446, 2005.

26. West, D.C., and Kumar, S. The effect of hyaluronate and its oligosaccharides on endothelial cell proliferation and monolayer integrity. Exp Cell Res 183, 179, 1989.

27. Locci, P., Marinucci, L.L., Martinese, D., and Becchetti, E. Transforming growth factor beta1-hyaluronic acid interaction. Cell Tissue Res 281, 317, 1995.

28. Macri, C., Silverstein, D., and Clark, A.F. Growth factor binding to the pericellular matrix and its importance in tissue engineering. Adv Drug Deliv Rev 59, 1366, 2007.

29. Marinucci, L.L., Stabellini, G., Belcastro, S., Becchetti, E., Balducci, C., Staffolani, N., and Locci, P. Biomembranes enriched with TGFbeta1 favor bone matrix protein expression by human osteoblasts in vitro. J Biomed Mater Res 63, 577, 2002. 
30. Kim, H.D., and Valentini, R.F. Retention and activity of BMP-2 in hyaluronic acid-based scaffolds in vitro. J Biomed Mater Res 59, 573, 2002.

31. Solchaga, L.A., Dennis, J.E., Goldberg, V.M., and Caplan, A.I. Hyaluronic acid-based polymers as cell carriers for tissue-engineered repair of bone and cartilage. J Orthop Res 17, 205, 1999.

32. Colnot, C., Romero, D.M., Huang, S., and Helms, J.A. Mechanisms of action of demineralized bone matrix in the repair of cortical bone defects. Clin Orthop Relat Res 435, 69, 2005.

33. Yee, A.J.M., Bae, H.W., Friess, D., Robbin, M., Johnstone, B., and Yoo, J.U. Augmentation of rabbit posterolateral spondylodesis using a novel demineralized bone matrixhyaluronan putty. Spine 28, 2435, 2003.

34. Morone, M.A., and Boden, S.D. Experimental posterolateral lumbar spinal fusion with a demineralized bone matrix gel. Spine 23, 159, 1998.
35. Yee, A.J.M., Bae, H.W., Friess, D., Robbin, M., Johnstone, B., and Yoo, J.U. Accuracy and interobserver agreement for determinations of rabbit posterolateral fusion. Spine 29, 1308, 2004.

Address correspondence to: William R. Walsh, Ph.D. Surgical and Orthopaedic Research Laboratories University of New South Wales Prince of Wales Hospital Randwick, NSW 2031

Australia

E-mail:w.walsh@unsw.edu.au

Received: January 5, 2010

Accepted: August 12, 2010

Online Publication Date: September 30, 2010 